\title{
Probing into the aging dynamics of biomass burning aerosol by using satellite measurements of aerosol optical depth and carbon monoxide
}

\author{
Igor B. Konovalov ${ }^{1}$, Matthias Beekmann ${ }^{2}$, Evgeny V. Berezin ${ }^{1}$, Paola Formenti ${ }^{2}$, Meinrat O. Andreae
}

$5 \quad{ }^{1}$ Institute of Applied Physics, Russian Academy of Sciences, Nizhniy Novgorod, Russia

${ }^{2}$ LISA/IPSL, Laboratoire Interuniversitaire des Systèmes Atmosphériques, UMR CNRS 7583, Université Paris Est Créteil

(UPEC) et Université Paris Diderot (UPD), France

${ }^{3}$ Biogeochemistry Department, Max Planck Institute for Chemistry, Mainz, Germany

${ }^{4}$ Scripps Institution of Oceanography, University of California San Diego, La Jolla, CA 92093, USA

10 Correspondence to: Igor B. Konovalov (konov@appl.sci-nnov.ru)

Here, we address a special situation where it turned out to be possible to elucidate some effects associated with aging of BB plumes without invoking the statistical analysis described in Sect. 2.4. We identified and considered such a situation in order to assess the adequacy of the photochemical age estimates defined by Eq. (4), as well as to get a tentative insight into the phenomena analyzed in Sect. 3.2 using a more general approach.

15 The analysis described in this section follows the same ideas as that presented in Konovalov et al. (2015) for a different region and period, except that here satellite measurements of aerosol and $\mathrm{CO}$ are used instead of ground-based (in-situ) measurements. In particular, we identified two sub-regions (see Fig. S1) referred below to as the "source" and "receptor" regions. The source region covers the locations of the major fires that occurred, in particular (but not only), on 21 July. Consideration of the dynamics of the BB aerosol hourly fields (see Fig. S2) simulated with CHIMERE indicates that BB plumes originating from fires that occurred on 21 July were transported in the northeast direction and passed, on 22 July, over the "receptor" region, where no large fires occurred on those two days. The BB emissions of particulate matter on 21 and 22 July are shown in Fig. S1a and Fig. S1b, respectively. Note that the aerosol emissions in the simulations discussed below were calibrated (see Sect. 2.1.3) by using the available MODIS AOD measurements only for the source region on 21 July; likewise, the emissions of gaseous species were calibrated against the respective IASI CO measurements.

25 The corresponding estimates of the BB aerosol photochemical age are shown in Fig. S1c and Fig. S1d. As expected, the photochemical age of $\mathrm{BB}$ aerosol in the receptor region is larger than in the source region. Specifically, while the photochemical age is estimated to be less than 10 hours for $82 \%$ of grid cells (provided with data) in the source region on 21 July, $80 \%$ of grid cells in the receptor region feature the photochemical age larger than 15 hours; the median values of the photochemical age are found to be 8.2 and 15.7 hours, respectively. It should be kept in mind that the photochemical age estimated in this study characterizes the column amounts of BB aerosol. As BB aerosol particles may have different ages at different altitudes, our vertically averaged photochemical ages might attenuate the contrast (with respect to the photochemical age) between the source and receptor regions. Fig. S3 presents the spatial distributions of AOD on 21 and 22 July according to the MODIS measurements and the CHIMERE simulations. A large BB plume featuring high AOD values $(>2)$ and passing over the receptor region on 22 July is clearly seen both in the measurements and simulations, while similar

35 AOD values are found mostly southwest, in the source region, and south (only in the measurements) from the receptor region on 21 July. The differences in the measured and simulated AOD values for 21 July indicate that emissions from fires that likely occurred somewhere south from the receptor region and north from Novosibirsk, Kemerovo and Achinsk have been missing (or strongly underestimated) in our simulations. However, the trajectories (see Fig. S4) calculated with the HYSPLIT model (http://ready.arl.noaa.gov/hysplit-bin) suggest that, due to the prevailing anticyclonic circulation around a high pressure area centered at about $82^{\circ} \mathrm{E}$ and $60^{\circ} \mathrm{N}$ on 21 and 22 July (according to data from the WRF-ARW model used in this study), the air masses over the locations of the probable missing aerosol sources were advected mostly 
southwestward, and so such sources could hardly contribute considerably to the BB plumes observed over the receptor region on 22 July.

The spatial distributions of the CO columns shown in Fig. S5 are qualitatively similar to those of AOD. This similarity confirms that $\mathrm{CO}$ and aerosol contributing to the major enhancements of CO columns and AOD over the study region originate from the same sources (that are most likely associated with wildfires). In addition to the above discussion of the sources and transport of BB plumes on 21 and 22 July, it can be noted that the differences between the distributions of CO columns on 21 and 22 July may be not only due to transport but also due to accumulation of air pollution from active fires over one day.

Figure S6 shows the relationships between BB fractions of CO columns $(\Delta v)$ and AOD $(\Delta \tau)$ in the source and receptor 10 regions according to the measurements and simulations. Note that the data shown in Fig. S6 were selected using the same criterion (based on the parameter $p_{\%}$ ) as in the analysis illustrated in Fig. 5a. The relationships between CO columns and AOD are analyzed here in the same way as the relationships between $\mathrm{CO}$ and $\mathrm{PM}_{10}$ concentrations in Konovalov et al. (2015, see Fig.7 therein) and are characterized quantitatively by linear regressions without intercept $(y=a x)$. Note that the regression coefficient $(a)$ evaluates the fractions involved in the right-hand part of Eq. (9); it can also be regarded as an analogue to

15 NEMR (see Introduction) in a special case where CO and aerosol column amounts are considered (under the assumption that changes in the mass extinction efficiency of BB aerosol can be disregarded) instead of $\mathrm{CO}$ and aerosol mass concentrations considered as in a more common case. Apart from the simulations performed with the "standard" aerosol scheme (that is, for the STN scenario), Fig. S6 shows the simulated data obtained from the VBS run of CHIMERE.

As expected, due to the calibration of BB emissions against satellite measurements, there is a close agreement between the 20 regression slopes calculated for the measurements and simulations in the source region. However, similar to Konovalov et al. (2015), we find considerable differences between the regression lines fitted to the measurement and simulation data for the receptor region. Specifically, the regression coefficient for the receptor region turns out to be a factor of 2 larger in the case with the measurement data than in the case with the simulated data obtained from the STN run. The use of the VBS scheme instead of the standard aerosol scheme in our model improves the agreement between the measurements and simulations to some extent (a value of $a$ for the VBS run is about $15 \%$ larger compared to the one for the STN run), but the difference between the slopes of the linear fits for the measurements and simulations still remains significant. Note that Konovalov et al. (2015) found that the use of the VBS scheme instead of the standard one to simulate near surface concentrations of BB OA resulted in a more substantial increase (specifically, by $47 \%$ ) of the regression coefficient than in this study, while the differences between the regressions fitted to the measurement data and to the simulations performed with the VBS scheme were not statistically significant. Presumably, the difference between the results of the VBS and STN runs is relatively small in the case addressed here partly because BB aerosol mass concentration was integrated over the vertical dimension (so, depending on vertical and horizontal transport patterns, a vertical column of BB aerosol might include aerosol particles having very different photochemical ages). This could also be partly because BB aerosol in the source region, although being relatively "fresh" compared to the aerosol in the receptor region, could still undergo considerable atmospheric processing (as evidenced by its median photochemical age of 8.6 hours).

The differences between the linear fits to the measurement data and simulations in the receptor region indicate that transport of BB plumes from the source region to the receptor region could be associated with a major increase of the optical depth of actual BB aerosol with respect to the simulated AOD values (especially from the STN run). Furthermore, such an increase can be interpreted as a manifestation of BB aerosol aging processes that are not properly taken into account in our model.

40 The increase of AOD could be not only due to an enhancement of the aerosol mass column density relative to the CO columns, but also due to an increase in the mass extinction efficiency which was assumed to be constant in our simulations. It should be noted, however, that apart from effects of aging processes, the inconsistencies between the measurements and 
simulations in the receptor region can, in principle, be caused by some other reasons, e.g., temporal variations in the actual emission factors or errors in the modeled vertical distributions of CO. Although such reasons are, in our opinion, less probable, they still cannot be definitely ruled out. Therefore, the results presented above should be regarded as evidence but not as a proof of an important role played by aging processes in the evolution of $\mathrm{BB}$ aerosol in the region and period 5 considered.

The results shown in Fig. S6 at least do not contradict to the results of the more general analysis (see Sect. 3.2 and Fig. 5a) that reveal an increase in the ER values within the first 15 hours of atmospheric processing of BB aerosol. Most of the data points (29 out of 32) corresponding to the source region on 21 July fall into the first and second bins $\left(t_{a}<11.2 \mathrm{~h}\right)$ of the photochemical age values based on concentration of the aging tracer $T_{1}$, while most of the data points (12 out of 20)

10 corresponding to the receptor region on 22 July fall into the third bin $\left(t_{a} \in[11.2 ; 16.3]\right)$; the rest of the data points for the receptor region belong to the forth bin $\left(t_{a} \in[16.3 ; 21.4]\right)$. Accordingly, the increase of AOD with respect to CO columns in the episode considered here could contribute to the increasing part of the dependence shown in Fig. 5a. However, the results of the two kinds of analysis are hard to compare quantitatively, particularly because the data points corresponding to the episode considered here provide only a minor contribution to the whole data set considered in Sect. 3.2 (specifically, the 15 first, second, third and forth photochemical age bins defined with the tracer $T_{1}$ contain 50, 439, 457, and 329 data points, respectively). 


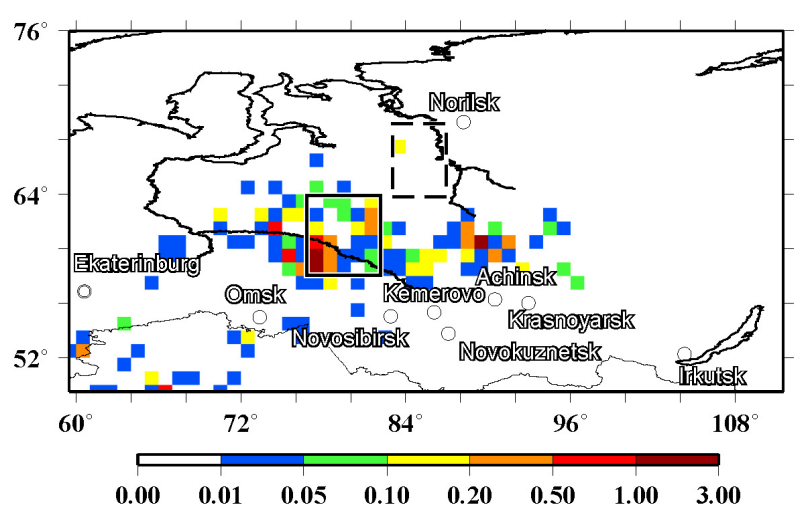

c

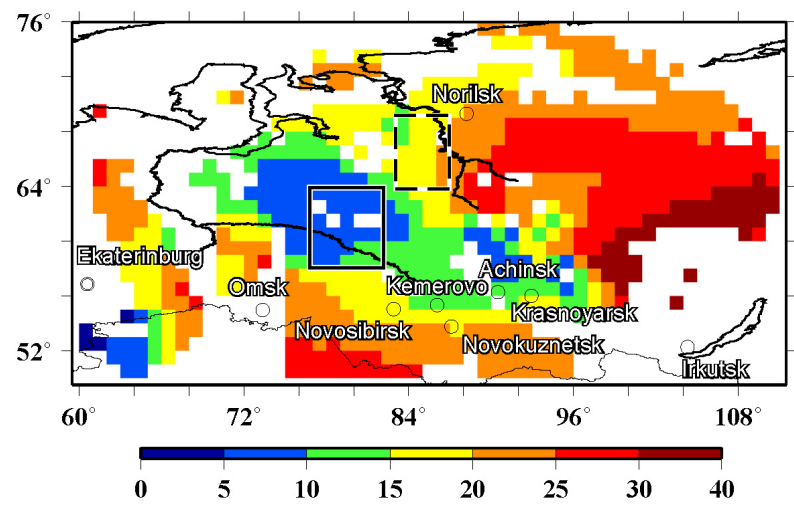

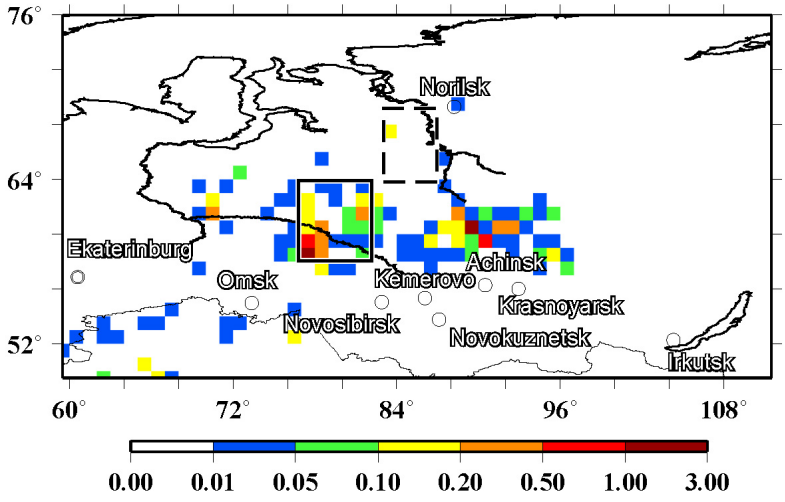

d

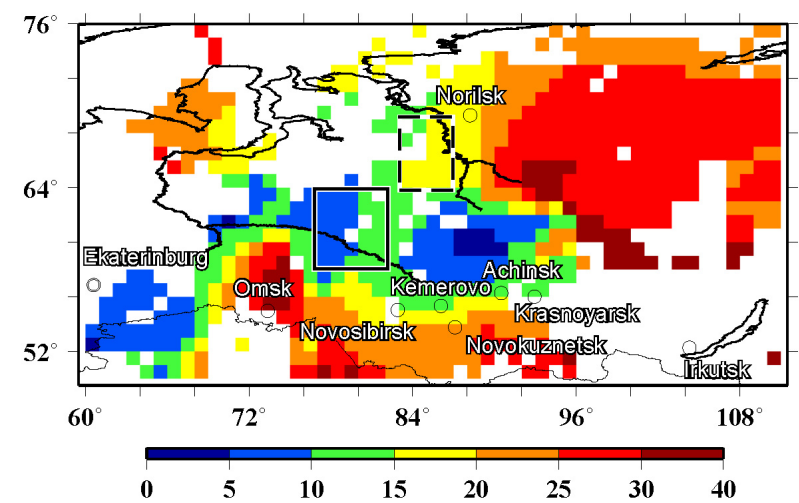

Figure S1: Spatial distributions of $(a, b)$ the emissions of particulate matter $\left(g \cdot \mathrm{m}^{2}\right)$ and $(c, d)$ the photochemical age (hours) of BB aerosol in the study region on (a,c) 21 and (b,d) 22 July 2012. Rectangles depicted with solid and dashed lines indicate the "source" and "receptor" regions. Note that the photochemical age was calculated using the tracers $T_{0}$ and $T_{1}$ (and is shown) only for those grid cells where the measurement AOD and CO data matching the simulations were available and BB emissions contributed significantly to both CO columns and AOD (according to the criteria given in Sect. 2.1.3). 

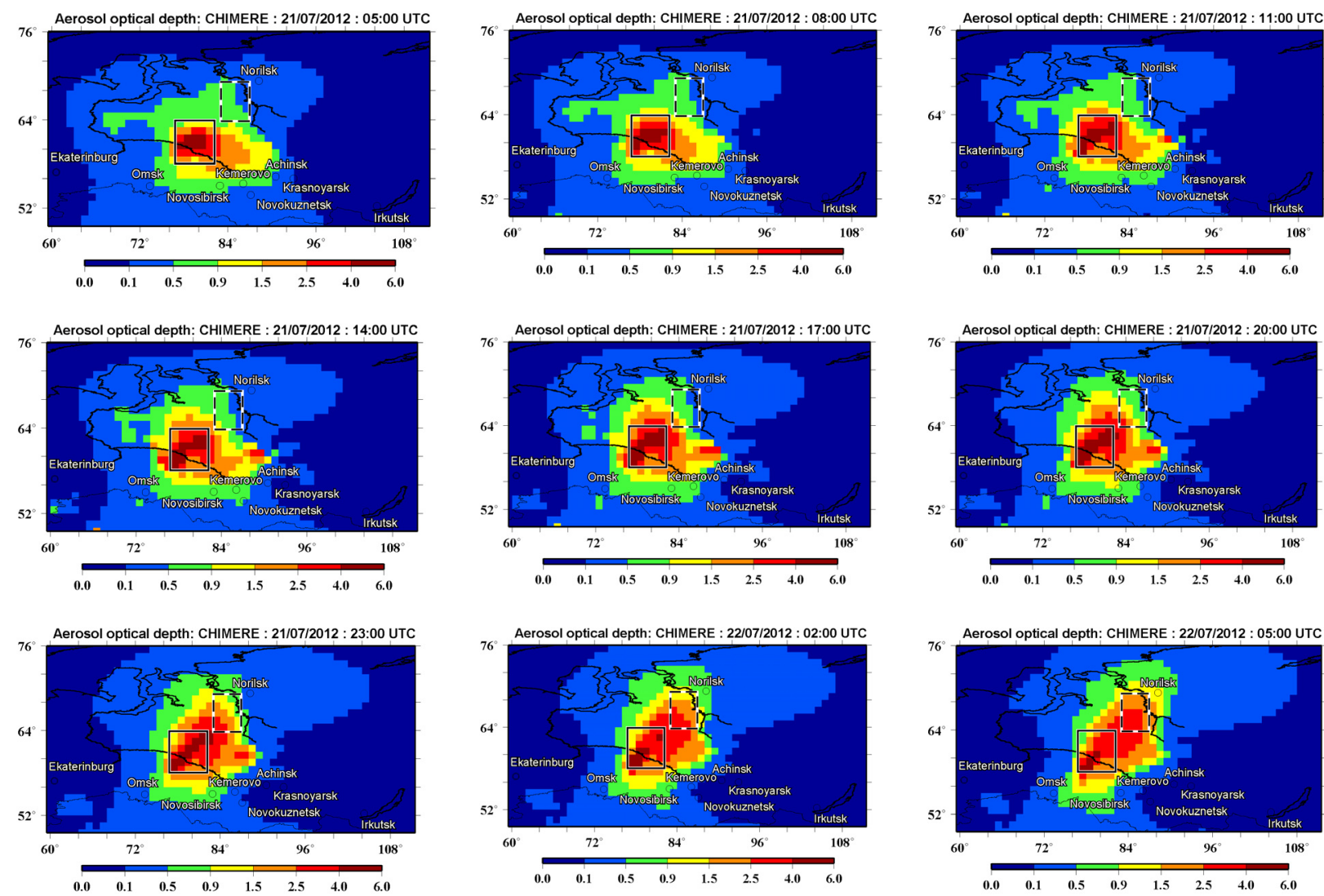

Figure S2: "Snapshots" of the spatial distribution of AOD in the period from 05:00 UTC July 21 to 05:00 UTC July 22 according to the CHIMERE simulations. 

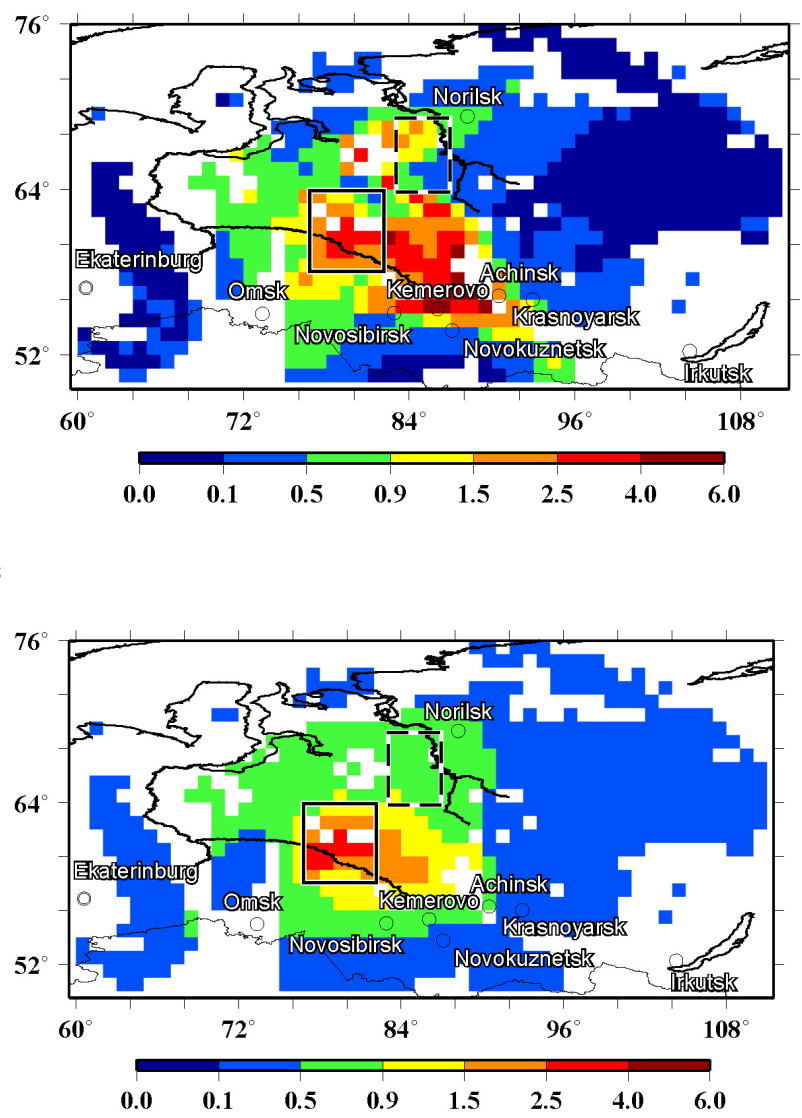

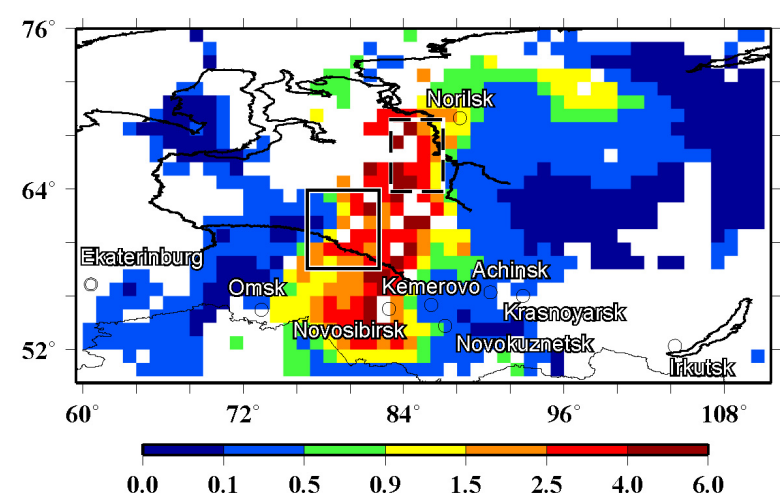

d

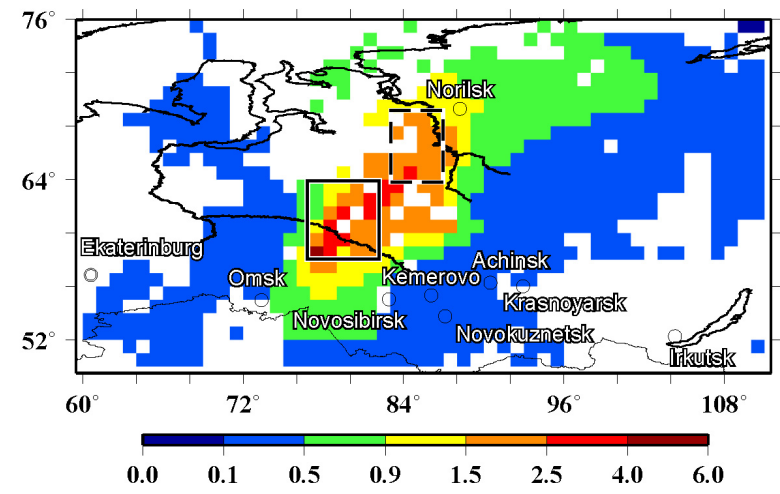

Figure S3: The spatial distributions of AOD on $(a, c) 21$ and $(b, d) 22$ July according to (a,b) the MODIS measurements and (c,d) the CHIMERE simulations. 


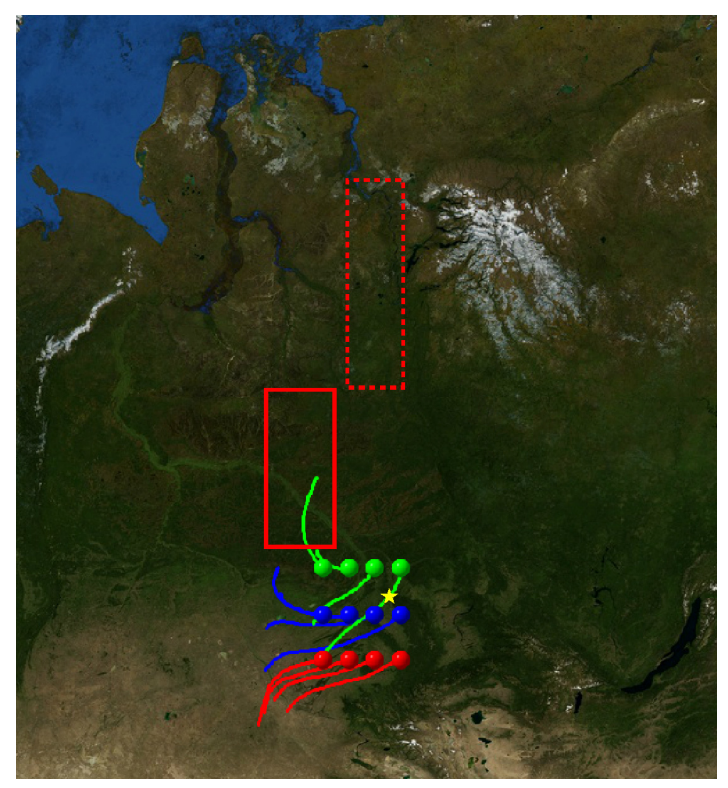

Figure S4: The HYSPLIT 24h forward trajectories starting from several locations (at altitude of $1000 \mathrm{~m}$ ) on 21 July 2012 at 6:00 UTC ( 11:30 LST). A yellow asterisk indicates the location of the Kemerovo city. The red rectangles depicted by solid and dotted curve indicate the source and receptor regions, respectively. 

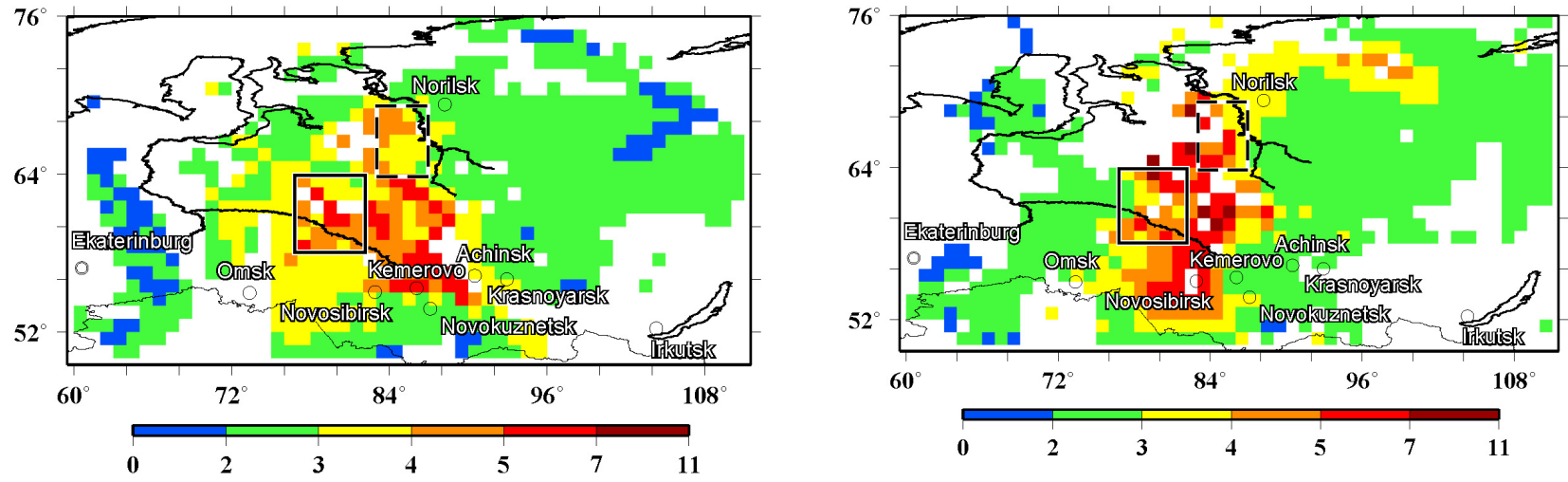

c

d
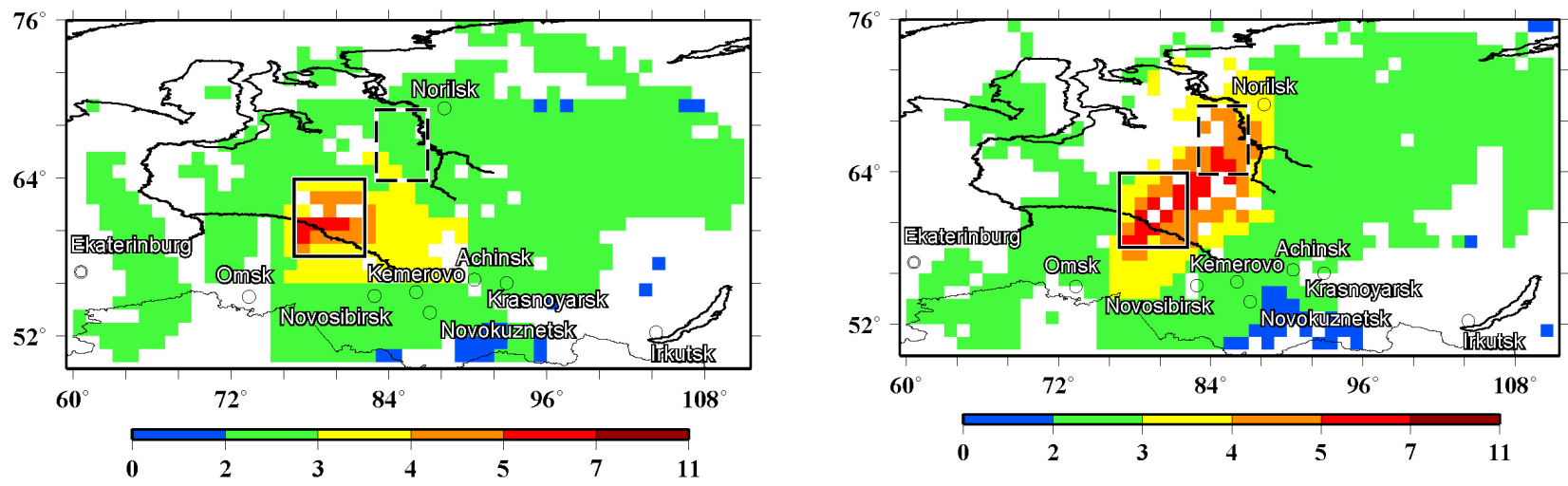

Figure S5: The spatial distributions of the total CO column amounts $\left(10^{18}\right.$ molecules $\left.\mathrm{cm}^{-2}\right)$ on (a,c) 21 and (b,d) 22 July according to $(a, b)$ the IASI measurements and $(c, d)$ the CHIMERE simulations. 

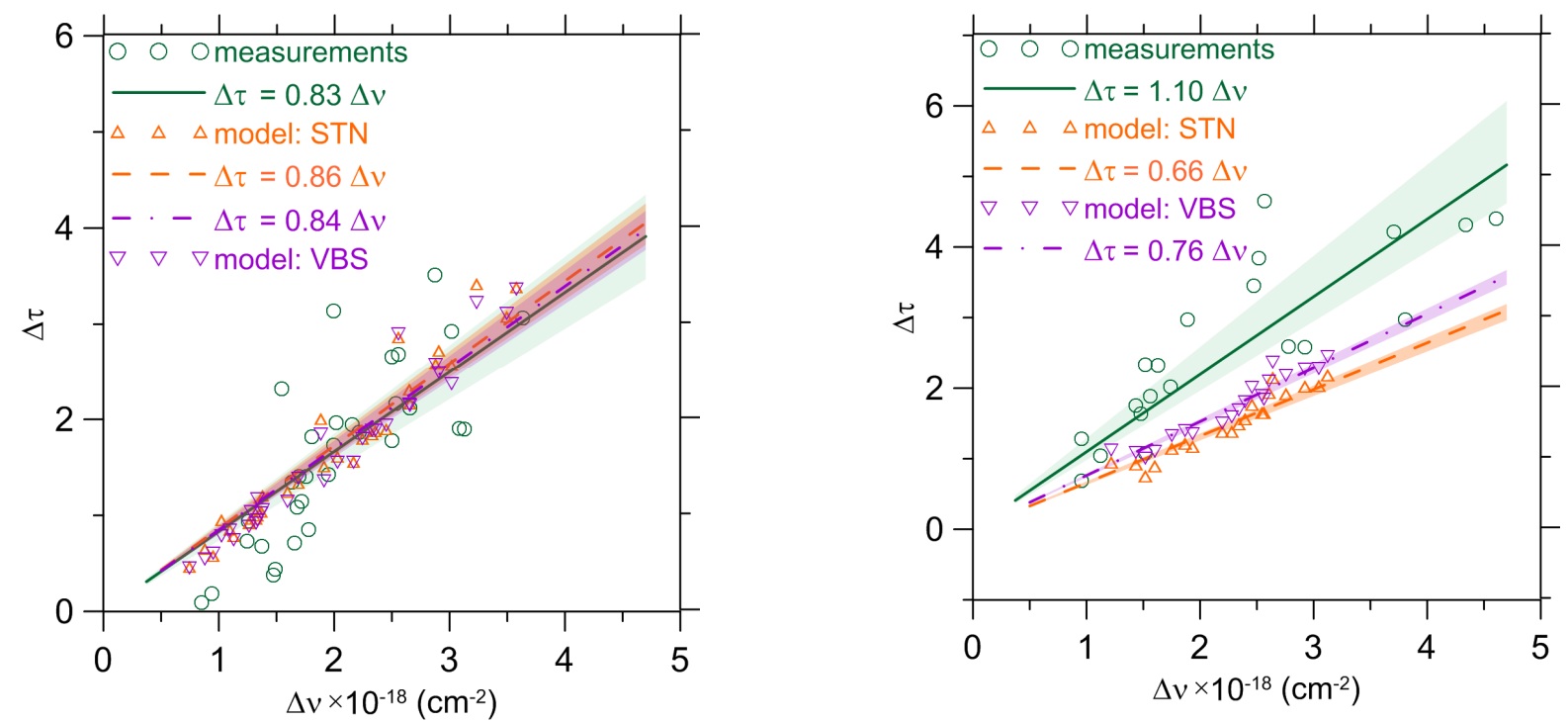

Figure S6: The relationships between BB fractions of (a) CO columns ( $\Delta v)$ and (b) AOD ( $\Delta \tau)$ in (a) the source region on 21 July and (b) in the receptor region on 22 July 2012 according to the satellite (MODIS and IASI) measurements (see green lines) and the simulations for the STN (orange dashed lines) and VBS (purple dash dot lines) configurations. The source and receptor regions are shown in Fig. S1. The relationships are fitted by means of the linear regression "through the origin". The regression coefficient values are indicated in the figure legends. Shaded areas depict the confidence intervals evaluated for the 95th percentile confidence level. 